\title{
First clinical use of a novel hypothermic storage system for a long-distance donor heart procurement
}

\author{
Noritsugu Naito, MD, PhD, Masaki Funamoto, MD, PhD, Richard N. Pierson, MD, and
}

David A. D’Alessandro, MD, Boston, Mass

\author{
From the Department of Cardiac Surgery, Massachusetts General Hospital, Boston, Mass. \\ Disclosures: Authors have nothing to disclose with regard to commercial support. \\ Received for publication April 7, 2019; revisions received May 22, 2019; accepted for publication May 31, 2019; \\ available ahead of print Aug 13, 2019. \\ Address for reprints: David A. D'Alessandro, MD, Department of Cardiac Surgery, Massachusetts General Hos- \\ pital, 55 Fruit St, Boston, MA 02114 (E-mail: DADALESSANDRO@mgh.harvard.edu). \\ J Thorac Cardiovasc Surg 2020;159:e121-3 \\ $0022-5223 / \$ 36.00$ \\ Copyright (C) 2019 by The American Association for Thoracic Surgery \\ https://doi.org/10.1016/j.jtcvs.2019.05.085
}

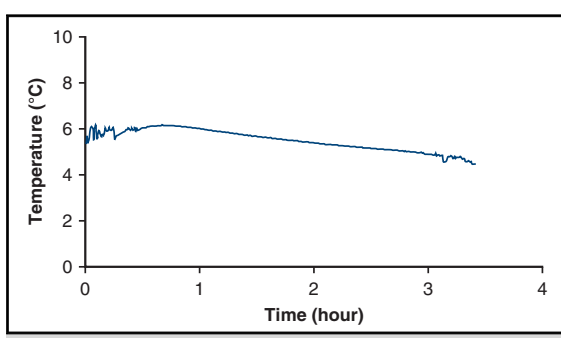

Organ temperature was maintained within a narrow range throughout the transportation.

Central Message

By maintaining donor heart temperature within an optimal range, donor hearts may tolerate longer ischemic times, reducing the risk of primary graft failure.

See Commentary on page e125.
Current cold storage (CS) heart preservation technique is associated with wide fluctuation of organ temperature and may result in freeze injury. The Paragonix SherpaPak (SherpaPak-CTS; Paragonix Technologies, Braintree Mass) cardiac transport system is a single-use disposable device designed for static hypothermic donor heart preservation and was recently approved in the United States for clinical use. We report the first clinical use of the SherpaPak-CTS for the human heart to transport a distantly procured heart from a brain-dead donor.

\section{CASE REPORT}

The recipient was a 65-year-old man with history of type 2 diabetes, atrial fibrillation, and ischemic cardiomyopathy, resulting in end-stage heart failure. The patient was listed for heart transplant, and a suitable donor became available approximately 1 month after the listing. The donor was a female patient in her thirties of similar height and weight to the recipient. She was hepatitis $\mathrm{C}$ antibody-seropositive, with negative results of nucleic acid amplification testing. The donor hospital was more than 1100 miles from the recipient institution, so a prolonged ischemic time was anticipated.

For the procurement, the SherpaPak-CTS was selected. Before donor aortic crossclamp, 3L cold standard Belzer UW solution (Bridge to Life Ltd, Columbia, SC) was decanted into the organ canister. The donor heart was recovered in a standard fashion with $2 \mathrm{~L} \mathrm{UW}$ solution in situ. A patent foramen ovale was closed on the back table. The heart connector was attached to the ascending aorta with umbilical tape (Video 1). The heart was anchored to the organ canister and preserved in the shipper (Figure 1). The donor heart temperature was maintained at $5.51^{\circ} \mathrm{C} \pm 0.46^{\circ} \mathrm{C}$ (mean $\pm \mathrm{SD}$ ) throughout transportation (Figure 2). Transportation time within the shipper was 205 minutes, and the total ischemic time was 312 minutes.

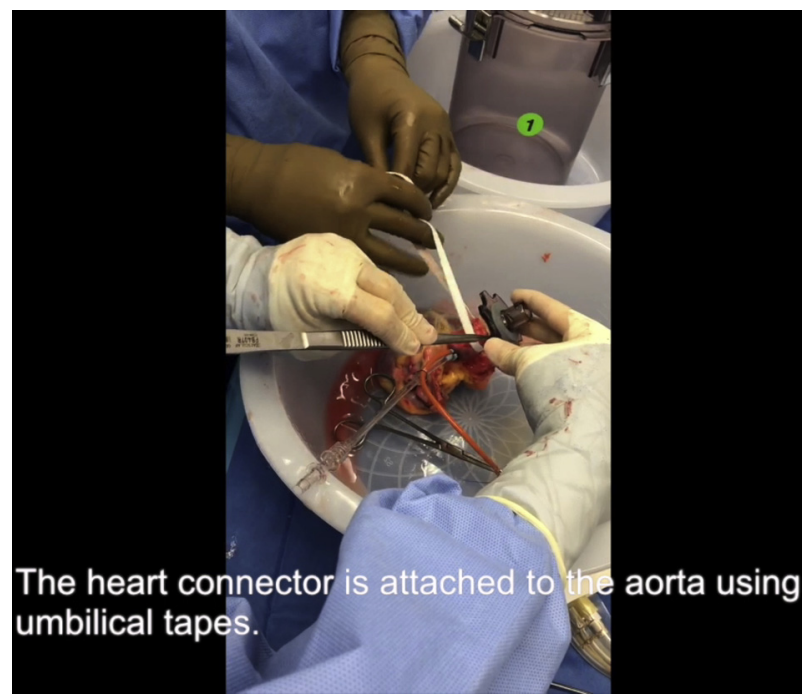

VIDEO 1. A video of the donor heart preservation technique. Video available at: https://www.jtcvs.org/article/S0022-5223(19)31352-2/fulltext. 

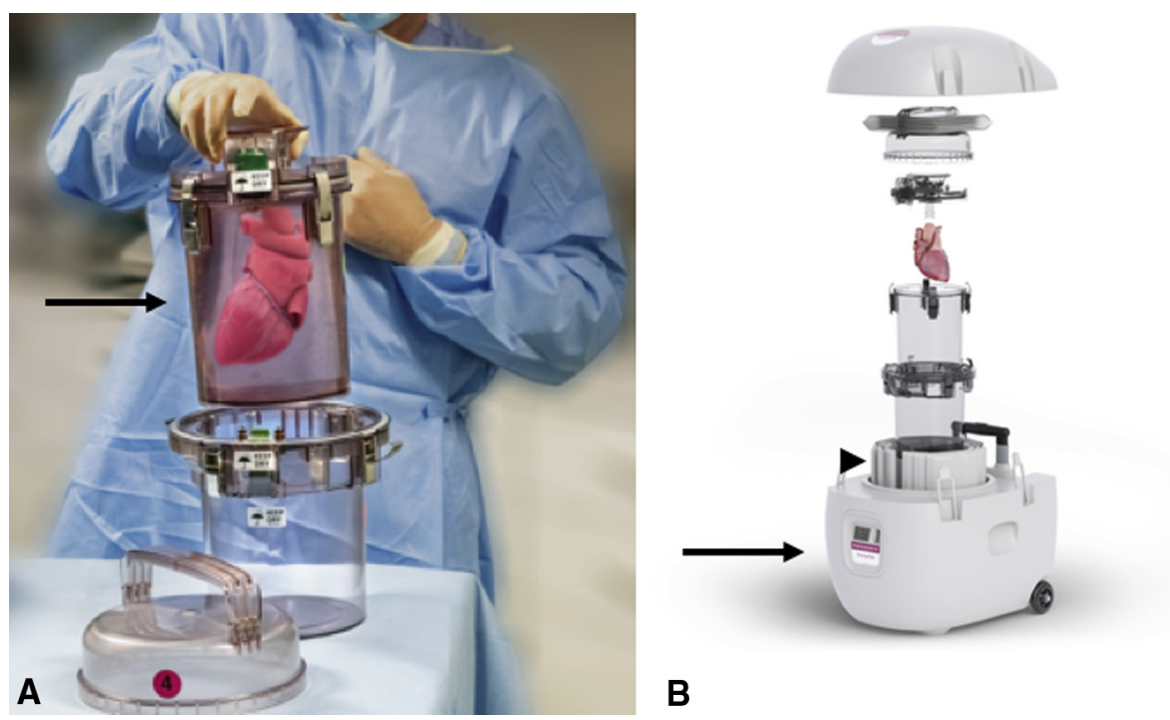

FIGURE 1. A schematic images of Paragonix SherpaPak-CTS. A, The donor heart is fully immersed in preservation solution in the inner cannister (arrow). The canister has real-time temperature sensor. The inner canister is placed in the outer canister. B, All the materials are for single use. Two rigid sterile canisters are surrounded by cold ice packs, engineered for high latent heat storage in the $4^{\circ} \mathrm{C}$ to $8^{\circ} \mathrm{C}$ range (arrowhead). The canisters and ice packs are placed in the insulated shipper (arrow), which provides triple barrier protection of organs.

The recipient operation was done uneventfully. Cardiopulmonary bypass was weaned with low-dose inotropes and vasopressors after 35 minutes of reperfusion. The recipient was extubated within 7 hours after the operation. Two episodes of tonic-clonic seizure were observed on postoperative day (POD) 0. Imaging studies showed no evidence of hemorrhage or embolism. No further seizures were observed.

Although the heart function was well preserved, inotropes and vasopressors were slowly weaned and discontinued by POD 4 . The recipient's postoperative course was complicated by acute kidney injury, with peak serum creatinine of $2.18 \mathrm{mg} / \mathrm{dL}$ (baseline, $1.4 \mathrm{mg} / \mathrm{dL}$ ), which recovered without dialysis. He was discharged from the intensive care unit to the step-down floor on POD 6. The second biopsy of the heart, on POD 14, showed grade $3 \mathrm{~A} / 2 \mathrm{R}$ rejection

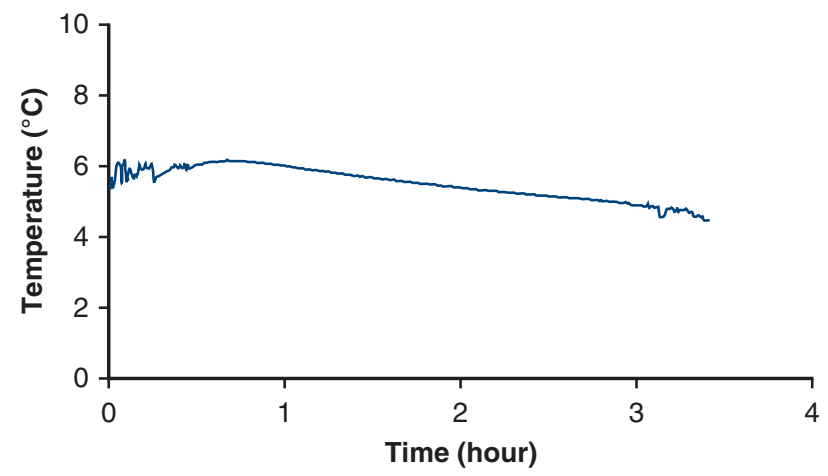

FIGURE 2. Temperature changes during transportation. Organ temperature was maintained within a narrow range throughout the transportation. requiring 3 days of steroid pulse therapy. The patient was discharged home on POD 17 and continues to do well in follow-up. Transthoracic echocardiography before discharge showed good biventricular function (left ventricular ejection fraction, $72 \%$ ).

\section{DISCUSSION}

Current CS technique for donor heart preservation limits the total ischemic time to 4 to 6 hours, and longer periods of ischemia are known to adversely affect survival. ${ }^{1}$ In addition, the organ temperatures during transportation can decrease to less than $0^{\circ} \mathrm{C}$ in long transportation, ${ }^{2}$ resulting in frost injury and uneven cooling. SherpaPak-CTS is designed to maintain donor heart temperature uniformly within the range of $4^{\circ} \mathrm{C}$ to $8^{\circ} \mathrm{C}$. Preclinical study with pig hearts showed that the device was able to maintain temperature within this range for longer than 12 hours. ${ }^{3}$ SherpaPak-CTS is the only commercially available static hypothermic heart transportation device cleared by US Food and Drug Administration and CE marked. The device is a reasonable alternative to $\mathrm{CS}$, with a potential benefit when longer transport times are expected. The clinical team easily adopted this technique after minimal device training and judged the system to be simple and effective in maintain the organ within a narrow temperature range.

Recently, there has been growing interest in pulsatile, normothermic ex vivo heart perfusion for marginal donor allografts. ${ }^{4}$ Although such a technique has been shown to be noninferior to $\mathrm{CS},{ }^{5}$ the preparation of this device takes longer, the management is more complicated, and the 
materials are considerably more expensive than the SherpaPak system, and it is an unlikely substitute for the CS method in routine transplantation. This report describes the first clinical use of a novel storage system. In the case presented here, the SherpaPak-CTS adequately preserved the donor organ, resulting in normal function despite an ischemic time beyond 5 hours. By maintaining donor heart temperature within an optimal range, donor hearts may tolerate longer ischemic times, reducing the risk of primary graft failure. Further investigation is needed to ascertain whether this method provides superior cardiac protection to that of the current CS method and whether the magnitude of such benefit justifies the increased equipment cost.

\section{References}

1. Trivedi JR, Cheng A, Ising M, Lenneman A, Birks E, Slaughter MS. Heart transplant survival based on recipient and donor risk scoring: a UNOS database analysis. ASAIO J. 2016;62:297-301.

2. Horch DF, Mehlitz T, Laurich O, Abel A, Reuter S, Pratschke H, et al. Organ transport temperature box: multicenter study on transport temperature of organs. Transplant Proc. 2002;34:2320.

3. Michel SG, LaMuraglia GM, Madariaga MLL, Anderson LM. Innovative cold storage of donor organs using the Paragonix SherpaPak devices. Heart Lung Vessel. 2015:7:246-55.

4. ClinicalTrials.gov. International EXPAND Heart Pivotal Trial (EXPANDHeart) Available at: http://clinicaltrials.gov/ct2/show/NCT02323321. Accessed March $28,2019$.

5. Ardehali A, Esmailian F, Deng M, Soltesz E, Hsich E, Naka Y, et al; PROCEED I trial investigators. Ex-vivo perfusion of donor hearts for human heart transplantation (PROCEED II): a prospective, open-label, multicentre, randomized noninferiority trial. Lancet. 2015;385:2577-84. 